\title{
Traffic signal control based on adaptive neural-fuzzy inference system applied to intersection
}

\begin{abstract}
Adaptive Neural-Fuzzy Inference System (ANFIS) that integrates the best features of fuzzy systems and neural networks has been widely applied in many areas. It can be applied to synthesize controllers, which are able to tune the fuzzy control system automatically, and models that learn from past data to predict future behavior. The aim of this research is to develop an ANFIS traffic signals controller for multilane intersection in order to ease traffic congestions at traffic intersections. The new concept to generate sample data for ANFIS training is introduced in this research. The sample data is generate based on fuzzy rules and can be analysed using tree diagram. This controller is simulated on multilane traffic intersection model developed using $\mathrm{M} / \mathrm{M} / 1$ queuing theory and its performance in terms of average waiting time, queue length and delay time are compared with traditional controllers and fuzzy controller. Simulation result shows that the average waiting time, queue length, and delay time of ANFIS traffic signal controller are the lowest as compared to the other three controllers. In conclusion, the efficiency and performance of ANFIS controller are much better than that of fuzzy and traditional controllers in different traffic volumes.
\end{abstract}

Keyword: ANFIS controller; Decision module; Green phase module; Multilane intersection; Next phase module 